\title{
PSMA-PET for the assessment of metastatic hormone-sensitive prostate cancer volume of disease
}

Francesco Barbato ${ }^{1}$, Wolfgang P. Fendler ${ }^{1}$, Isabel Rauscher ${ }^{2}$, Ken Herrmann ${ }^{1}$, Axel Wetter ${ }^{3}$, Justin Ferdinandus ${ }^{1}$, Robert Seifert ${ }^{4}$, Michael Nader ${ }^{1}$, Kambiz Rahbar ${ }^{4}$, Boris Hadaschik ${ }^{5}$, Matthias Eiber ${ }^{2}$, Andrei Gafita ${ }^{2}$, Manuel Weber ${ }^{*}$

${ }^{1}$ Department of Nuclear medicine, University of Duisburg-Essen and German Cancer Consortium (DKTK)-University Hospital Essen, Essen, Germany.

${ }^{2}$ Klinikum Rechts der Isar, Department of Nuclear Medicine, Technical University of Munich, Munich, Germany.

${ }^{3}$ Institute of Diagnostic and Interventional Radiology and Neuroradiology, University of Duisburg-Essen and German Cancer Consortium (DKTK)-University Hospital Essen, Essen, Germany.

${ }^{4}$ University of Münster and Cancer Consortium (DKTK)-University Hospital Münster, Münster, Germany

${ }^{5}$ Department of Urology, University of Duisburg-Essen and German Cancer Consortium (DKTK)-

University Hospital Essen, Essen, Germany.

* corresponding author

\section{First author:}

Name: Francesco Barbato, M.D.

Mailing address: Hufelandstraße 55, 45147 Essen, Germany

Telephone number: +49 2017232032

Fax number: +49 2017235658

E-mail address: Francesco.barbato@uk-essen.de

\section{Corresponding author:}

Name: Manuel Weber, M.D.

Mailing address: Hufelandstraße 55, 45147 Essen, Germany

Telephone number: +49 2017232032

Fax number: +49 2017235658

E-mail address: manuel.weber@uk-essen.de

\section{Keywords:}

PSMA, prostate cancer, mHSPC, metastasis-directed treatment, CHAARTED

\section{Running title:}

PSMA-PET-based stratification of mHSPC

Word count: 2328 


\begin{abstract}
:
Conventional imaging low-(LVD) versus high-volume disease (HVD) are associated with survival in metastatic hormone-sensitive prostate cancer (mHSPC) according to CHAARTED and STAMPEDE trials. We propose a compatible quantitative PSMA-PET framework for disease volume assessment in mHSPC.
\end{abstract}

Methods: Three PET centers screened their PSMA-PET database for mHSPC patients. CT versus PSMA-PET stage, lesion number, and classification of LVD vs. HVD were determined by one blinded reader; PSMA-positive tumor volume (PSMA-TV) was quantified semiautomatically.

Results: 85 CT-based CHAARTED-LVD and 20 CT-based CHAARTED-HVD patients were included. A PSMA-TV of $\sim 40 \mathrm{ml}$ was the optimal cutoff between CT-based CHAARTED-LVD (non-unifocal) and HVD (non-M1c) (AUC 0.86). Stratification into PET-LVD (unifocal or oligometastatic/disseminated $<\sim 40 \mathrm{~mL}$ ) and PET-HVD (oligometastatic/disseminated $\geq \sim 40 \mathrm{~mL}$ or M1c) had 13\% misalignment with CHAARTED criteria.

Conclusions: PSMA-PET criteria with volume quantification deliver comparable LVD/HVD discrimination with additional subgroups for unifocal, oligometastatic and disseminated disease, critical for guidance of targeted or multimodal therapy. 


\section{INTRODUCTION}

In the CHAARTED study (Chemo Hormonal Therapy versus Androgen Ablation Randomized Trial for Extensive Disease in Prostate Cancer) metastatic hormone-sensitive prostate cancer (mHSPC) patients with high volume disease (HVD) particularly benefitted from combined chemohormonal treatment (1). In addition, the STAMPEDE trial has shown improved failure-free survival in metastatic prostate cancer patients with low volume disease (LVD) undergoing radiotherapy of the primary (2). Beyond their primary goals, CHAARTED and STAMPEDE underline the importance of tumor burden assessment for the prediction of treatment response. In both trials, tumor burden was assessed based on conventional imaging, such as computed tomography $(\mathrm{CT})$, magnetic resonance imaging and bone scan. However, novel prostate-specific membrane antigen-ligand positron-emission tomography (PSMA-PET) demonstrates superior accuracy for prostate cancer localization at initial diagnosis, biochemical recurrence and for advanced disease (3-5). PSMA-PET further offers automatic disease volume quantification (6). Superior imaging properties, growing availability, and novel applications such as guidance of targeted therapy, necessitate an updated framework for mHSPC tumor volume assessment.

PSMA-PET accuracy improves selection of treatment pathways and guidance of local and systemic therapy. The potential of metastasis-directed treatment for disease control, which may delay the need for systemic treatment, has recently been shown (7).

The aim of this retrospective study is to define novel PSMA-PET mHSPC disease volume criteria that are compatible with CHAARTED/STAMPEDE and offer improved assessment such as (semi-)automatic volume quantification and identification of unifocal to oligometastatic disease subgroups.

\section{MATERIALS AND METHODS}

\section{Patients}

PSMA-PET databases of three high-volume PET centers (University Hospital Essen, Technical University of Munich, University Hospital Münster) were screened for prostate cancer patients meeting the following inclusion criteria which were adapted from the criteria in the CHAARTED study:

(1) Metastases on CT 
(2) Hormone-sensitive prostate cancer, defined as either

- No history of ADT or

- Concurrent ADT for $\leq 120$ days without progression or prior ADT (duration: $\leq 24$ months, no progression within 12 months of completion)

The retrospective analysis was approved by the local Ethics committee (University DuisburgEssen, faculty of medicine; protocol number: 18-8094-BO) and requirement of study-specific consent was waived.

\section{Image acquisition}

All PET images were acquired on a Siemens Biograph mCT after the mean administration of 128 (interquartile range (IQR): 93.8-148.3) MBq ${ }^{68} \mathrm{Ga}-\mathrm{PSMA} 11$ (n=79), or 333 (IQR: 270.5-386.9) MBq ${ }^{18}$ F-rhPSMA ( $\mathrm{n}=17$ ), or 328 (IQR: 292.5-356.0) MBq ${ }^{18} \mathrm{~F}-\mathrm{PSMA} 1007(\mathrm{n}=9)$ and an uptake interval of 70 (IQR: 59-80) minutes.

\section{Image analysis}

PET/CT and the corresponding CT dataset were anonymized separately and read by one blinded central reader with at least 2 weeks between PET/CT and CT reading sessions using OsiriX MD (Pixmeo SARL, Switzerland). Pathological findings were assessed for five regions (prostate bed, pelvic lymph nodes, extrapelvic lymph nodes, bones, and visceral) including number of lesions and subregions involved in accordance with PROMISE molecular imaging TNM classification (8). In accordance with CHAARTED, HVD was defined as the presence of visceral metastases and/or $\geq 4$ bone metastases ( $\geq 1$ beyond the vertebral bodies or the pelvis) based on the CT dataset (1). PSMA-positive tumor volume (PET volume) segmentation was carried out semiautomatically using qPSMA software (6), as follows:

Tumor lesions were localized on CT and segmented on PET with a free-hand isocontour using a standardized uptake value higher than 3.0 (bone tissue) or higher than the mean liver uptake (soft-tissue) as thresholds, respectively.

\section{Statistical analysis}

Descriptive statistics are provided. To assess PET volume optimal cut-off for CT-based CHAARTED HVD vs. LVD a Receiver operator characteristic (ROC) area under the curve 
(AUC) with Youden's J index was employed after excluding patients with unifocal or M1cdisease. The statistical analysis was performed using SPSS software version 26.0 (BM Corp., Armonk, N.Y., USA). Comparison of ROC curves was performed following the methodology of Hanley \& McNeil (9) using MedCalc Statistical Software version 19.7.1 (MedCalc Software, Ostend, Belgium).

\section{RESULTS}

\section{Patients' characteristics}

105 patients were included; the median age was 68.2 (IQR: 65-73) years, median PSA was 2.6 (IQR: $0.9-7.9) \mathrm{ng} / \mathrm{mL}$. Gleason Score was $\geq 8$ in 56/105 (53\%) patients. Primary treatment was radical prostatectomy in 78/105 (74\%) patients; 5/105 (5\%) patients had undergone primary radiotherapy and 16/105 (15\%) either had prior or concurrent treatment with ADT. A subgroup of 40 of 105 (38\%) patients had 3-year follow-up since PSMA-PET. Of these, 5 patients $(12.5 \%$ ) died from prostate cancer (Figure 1). Of these patients, four had CT-based CHAARTED and PET HVD and one CT-based CHAARTED LVD and PET HVD. 65 of 105 (62\%) patients had less than 3-year follow-up and were alive.

Supplemental Table 1 gives an overview of patients' characteristics.

\section{CT disease extent}

Among 105 patients, one (1\%) had pelvic only, five (5\%) extrapelvic nodal only (M1a), eight (8\%) any visceral (M1c), and 91 (87\%) any bone involvement. 85 patients had CHAARTED LVD and 20 HVD ( 8 with visceral metastases, 12 with extensive bone involvement).

\section{PSMA-PET disease extent}

Among 105 patients, one (1\%) had pelvic only, three (3\%) extrapelvic nodal only (M1a), ten $(10 \%)$ any visceral (M1c), and 91 (87\%) bone involvement without visceral metastases. Table 1 summarizes changes for CT versus PSMA-PET tumor extent on a lesion basis. When compared to CT findings, more lesions were found in 65/105 (62\%) of patients resulting in upstaging in 42/105 (40\%). Overall, 25/105 (24\%) patients had unifocal disease, 39/105 (37\%) oligometastatic disease, and 41/105 (39\%) multifocal disease on PSMA-PET. Supplemental Table 2 gives an overview of TNM stage migration. 
When formally applying CHAARTED criteria to PET, PSMA-PET led to hypothetical migration to CHAARTED HVD in 16/85 (19\%) patients due to the detection of additional bone metastases $(14 / 85,16.5 \%)$, or visceral metastases $(2 / 85,2 \%)$. To optimize alignment of CT-based CHAARTED versus PET-based volume of disease classification, PET volume was assessed.

\section{PET volume and PET HVD vs. LVD classification}

Mean PET volume was significantly higher in CT-based CHAARTED HVD vs. LVD patients (320.9 ml vs. $21.2 \mathrm{ml}$; p<0.001). ROC analysis showed an AUC of 0.86 for the PET volumebased classification of disease volume with $38.8 \mathrm{~mL}$ (termed $\sim 40 \mathrm{~mL}$ in the following) as the optimal cut-off value. Optimal cut-off was applied to define a PET volume of disease classification with lowest reasonably achievable misalignment (13\%) with CT-based CHAARTED criteria: PET-LVD (any unifocal; oligometastatic or disseminated $<\sim 40 \mathrm{~mL}$ ) versus PET-HVD (oligometastatic or disseminated $\geq \sim 40 \mathrm{~mL}$; any visceral) (Figure 1). An additional ROC analysis of only those patients, who had undergone ${ }^{68} \mathrm{Ga}-\mathrm{PSMA}$ PET, showed an AUC of 0.84 and confirmed the optimal cut-off value of $38.8 \mathrm{~mL}$. Comparison of both ROC curves did not show statistically significant differences $(\mathrm{p}=0.96)$.

PET versus CT-based volume of disease misalignment is detailed in Supplemental Table 3.

\section{DISCUSSION}

In this study with similar inclusion criteria to CHAARTED, PSMA-PET when compared with CT detected additional lesions in almost two-thirds of mHSPC patients leading to hypothetical CHAARTED LVD-to-HVD migration in about one-fifth of patients. PSMA-PET offers improvement by semi-automatic disease volume measurement. Implementation of a $\sim 40$ $\mathrm{mL}$ PET volume cut-off for patients with oligo- to disseminated metastatic disease was implemented in newly proposed PET volume of disease criteria with additional groups for unifocal and low volume oligometastatic disease. PET volume of disease criteria were aligned with current CT-based CHAARTED disease volume groups (13\% difference).

This study demonstrated high agreement of PSMA-PET and CT for the involved TNM regions in mHSPC patients. However, PSMA-PET detected significantly more lesions with quantifiable tumor volume (3-6). High accuracy enables visualization of unifocal or oligometastatic disease extent. PSMA-PET may aid in target volume delineation for metastasis-directed treatment, thus potentially delaying the need for systemic treatment, as previously assessed in the ORIOLE, 
STOMP, and SABR/COMET studies $(7,10,11)$. On the other hand, PSMA-PET detected disseminated disease in one of five patients with uni- or oligometastatic disease on conventional imaging. Upstaging aids accurate identification of candidates for systemic treatment and prevents standalone metastasis-directed treatment in cases where the clinical benefit is questionable.

In addition, PSMA-PET semi-automatic tumor volume assessment allows for reproducible classification of PET HVD vs. LVD. Using an optimized $40 \mathrm{~mL}$ cut-off PET volume of disease classification was largely compatible to CT-based CHAARTED criteria. Higher accuracy and rapidly growing availability of PSMA-PET necessitates revised disease volume definitions. However, novel PET criteria need to align with current evidence on prognostic groups within CHAARTED and STAMPEDE trials. Proposed PET criteria may allow for more accurate patient stratification in future clinical trials. PET subdivides LVD patients into those potentially eligible for metastasis-directed treatment (unifocal or oligometastatic disease) vs. those with low volume non-targetable disseminated disease (Figure 1).

Shortcomings of the study are the lack of correlational bone scans and missing long-term survival follow-up due to recent introduction of PSMA-PET. When compared to the CHAARTED trial, our study included a larger proportion of patients with recurrent prostate cancer after initial therapy and lower PSA values. This difference affects the comparability of results.

qPSMA tumor volume measurement has been validated using ${ }^{68} \mathrm{Ga}-\mathrm{PSMA}$ datasets. Therefore, caution is warranted when expanding this framework to patients staged with ${ }^{18} \mathrm{~F}$-labelled PSMAligands; however, inclusion of ${ }^{18}$ F-PSMA did not significantly impact optimal PET volume cutoff in our study.

\section{CONCLUSIONS}

Here we propose novel PET volume of disease criteria: PET-LVD (any unifocal; oligometastatic or disseminated $<\sim 40 \mathrm{~mL}$ ) versus PET-HVD (oligometastatic or disseminated $\geq \sim 40 \mathrm{~mL}$; any visceral). Novel PET criteria demonstrate acceptable alignment with CHAARTED/STAMPEDE groups but need to be validated in larger patient cohorts with survival follow-up.

\section{DISCLOSURES}

Francesco Barbato, Justin Ferdinandus, Axel Wetter, Michael Nader, Robert Seifert, Andrei Gafita, and Manuel Weber have nothing to disclose. Wolfgang P. Fendler is a consultant for 
Endocyte and BTG and received personal fees from RadioMedix outside of the submitted work. Ken Herrmann reports personal fees from Bayer SIRTEX, Adacap, Curium, Endocyte, IPSEN, Siemens Healthineers, GE Healthcare, Amgen, Novartis, and ymabs, personal fees and other from Sofie Biosciences, non-financial support from ABX, grants and personal fees from BTG, outside the submitted work. Kambiz Rahbar has received consultant fees from Bayer and ABX, lectureship fees from Janssen Cilag, Amgen, AAA and SIRTEX and travel expenses as unpaid member of the steering committee of the VISION trial. Boris Hadaschik reports advisory roles for ABX, Bayer, Lightpoint Medical, Inc., Janssen R\&D, Bristol-Myers-Squibb and Astellas; research funding from German Research Foundation, Janssen R\&D, Bristol-Myers-Squibb and Astellas; and travel support from AstraZeneca, Janssen R\&D and Astellas. Matthias Eiber reports an advisory role for Blue Earth Diagnostics, Point Biopharma, Telix and Janssen and patent application for rhPSMA.

\section{KEY POINTS}

QUESTION: Does PSMA-PET hold potential for improved tumor volume assessment over CT in patients with metastatic hormone-sensitive prostate cancer?

PERTINENT FINDINGS: PSMA-PET detects additional lesions in 62\% of patients with metastatic hormone-sensitive prostate cancer. Classification by virtue of quantified tumor volume and lesion number yields comparable results to conventional low vs. high-volume disease frameworks while providing a more accurate portrayal of true disease extent.

IMPLICATIONS FOR PATIENT CARE: The unparalleled accuracy of PSMA-PET allows for improved tumor volume assessment in metastatic hormone-sensitive prostate cancer and may facilitate the implementation of metastasis-directed treatment. 


\section{REFERENCES}

1. Sweeney CJ, Chen YH, Carducci M, et al. Chemohormonal Therapy in Metastatic HormoneSensitive Prostate Cancer. N Engl J Med. 2015;373:737-746.

2. Parker CC, James ND, Brawley CD, et al. Radiotherapy to the primary tumour for newly diagnosed, metastatic prostate cancer (STAMPEDE): a randomised controlled phase 3 trial. Lancet. 2018;392:2353-2366.

3. Fendler WP, Calais J, Eiber M, et al. Assessment of 68Ga-PSMA-11 PET Accuracy in Localizing Recurrent Prostate Cancer: A Prospective Single-Arm Clinical Trial. JAMA Oncol. 2019;5:856-863.

4. Fendler WP, Weber M, Iravani A, et al. Prostate-Specific Membrane Antigen Ligand Positron Emission Tomography in Men with Nonmetastatic Castration-Resistant Prostate Cancer. Clin Cancer Res. 2019;25:7448-7454.

5. Hofman MS, Lawrentschuk N, Francis RJ, et al. Prostate-specific membrane antigen PET-CT in patients with high-risk prostate cancer before curative-intent surgery or radiotherapy (proPSMA): a prospective, randomised, multicentre study. Lancet. 2020;395:1208-1216.

6. Gafita A, Bieth M, Kronke M, et al. qPSMA: Semiautomatic Software for Whole-Body Tumor Burden Assessment in Prostate Cancer Using (68)Ga-PSMA11 PET/CT. J Nucl Med. 2019;60:1277-1283.

7. Phillips R, Shi WY, Deek M, et al. Outcomes of Observation vs Stereotactic Ablative Radiation for Oligometastatic Prostate Cancer: The ORIOLE Phase 2 Randomized Clinical Trial. JAMA Oncol. 2020 .

8. Eiber M, Herrmann K, Calais J, et al. Prostate Cancer Molecular Imaging Standardized Evaluation (PROMISE): Proposed miTNM Classification for the Interpretation of PSMA-Ligand PET/CT. J Nucl Med. 2018;59:469-478.

9. Hanley JA, McNeil BJ. A method of comparing the areas under receiver operating characteristic curves derived from the same cases. Radiology. 1983;148:839-843.

10. Ost P, Reynders D, Decaestecker K, et al. Surveillance or Metastasis-Directed Therapy for Oligometastatic Prostate Cancer Recurrence: A Prospective, Randomized, Multicenter Phase II Trial. $J$ Clin Oncol. 2018;36:446-453.

11. Palma DA, Olson R, Harrow S, et al. Stereotactic ablative radiotherapy versus standard of care palliative treatment in patients with oligometastatic cancers (SABR-COMET): a randomised, phase 2, open-label trial. Lancet. 2019;393:2051-2058. 
Figure 1: mHSPC disease extent by CT-based CHAARTED vs. PET-based miTNM Volume criteria

mHSPC disease extent as stratified by CT using CHAARTED criteria and PET using tumor volume and miTNM. Criteria for combined (miTNM \& Volume) PET volume of disease assessment are shown. The dashed line indicates AUC-ROC-derived $\sim 40 \mathrm{~mL}$ cutoff-value between PET-LVD and PET-HVD. LVD=low-volume disease, HVD=high-volume disease. 3year overall survival is given for $n=40$ patients $(0$, alive; $\uparrow$, died; all other alive and 3 -years postPET not yet reached). ${ }^{*} n=1$ patient with CHAARTED HVD and PET LVD.

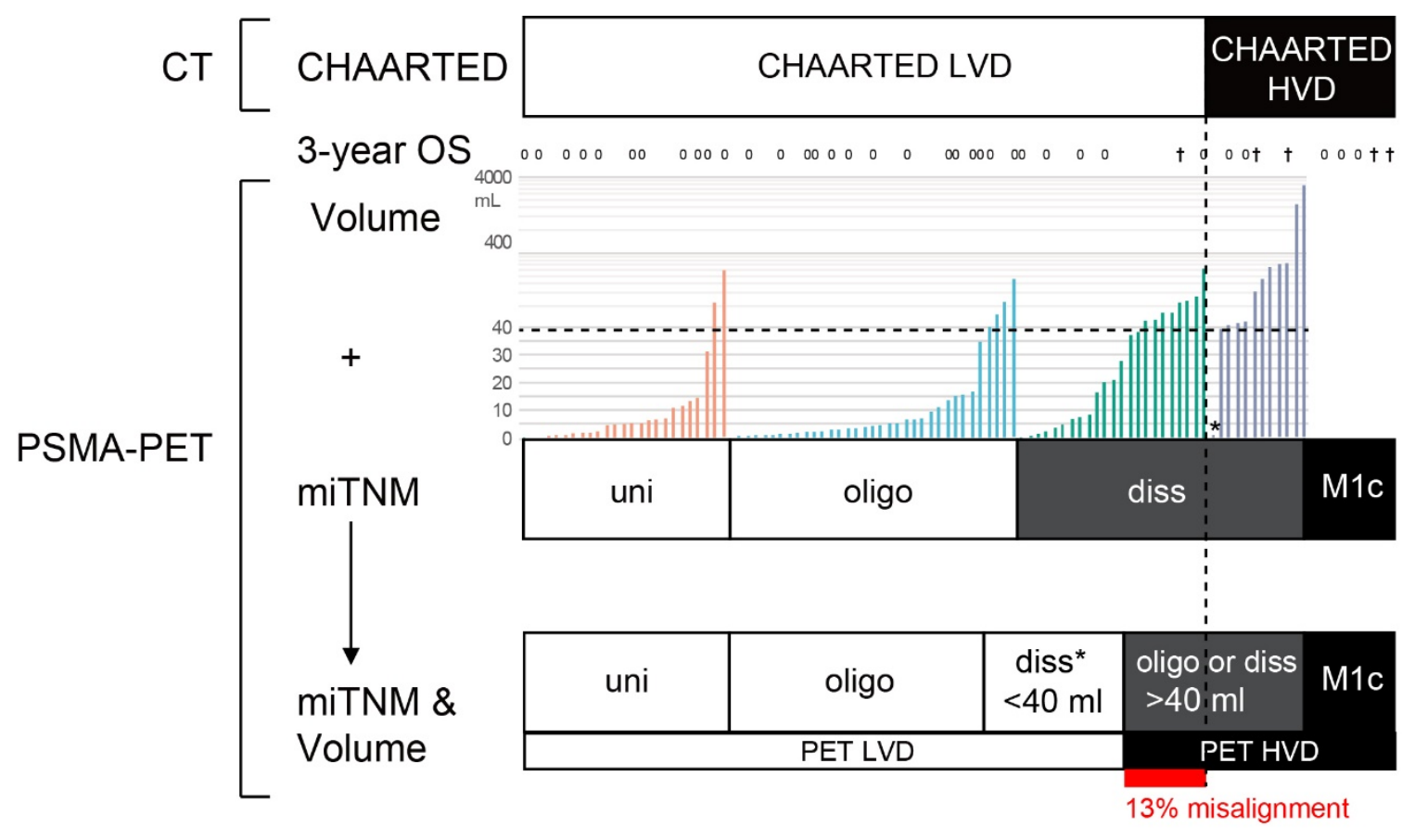


Table 1: mHSPC disease extent by computed tomography (CT) versus PSMA-PET.

\begin{tabular}{|c|c|c|c|c|c|}
\hline & \multicolumn{5}{c|}{ PSMA-PET } \\
\hline \multirow{3}{*}{ CT } & Stage n (\%) & unifocal & oligometastatic & disseminated & Any M1c \\
\cline { 2 - 7 } & unifocal & $\mathbf{2 5}(\mathbf{2 4})$ & $19(18)^{*}$ & $7(7)^{*}$ & $2(2)^{*}$ \\
\cline { 2 - 7 } & oligometastatic & 0 & $\mathbf{1 6 ( 1 5 )}$ & $14(13)^{*}$ & 0 \\
\cline { 2 - 7 } & disseminated & 0 & 0 & $\mathbf{1 4}(\mathbf{1 3})$ & 0 \\
\cline { 2 - 7 } & Any M1c & 0 & 0 & 0 & $\mathbf{8}(\mathbf{8})$ \\
\hline
\end{tabular}

*PET upstaging in 42 of $105(40 \%)$ patients.

\section{Graphical Abstract}

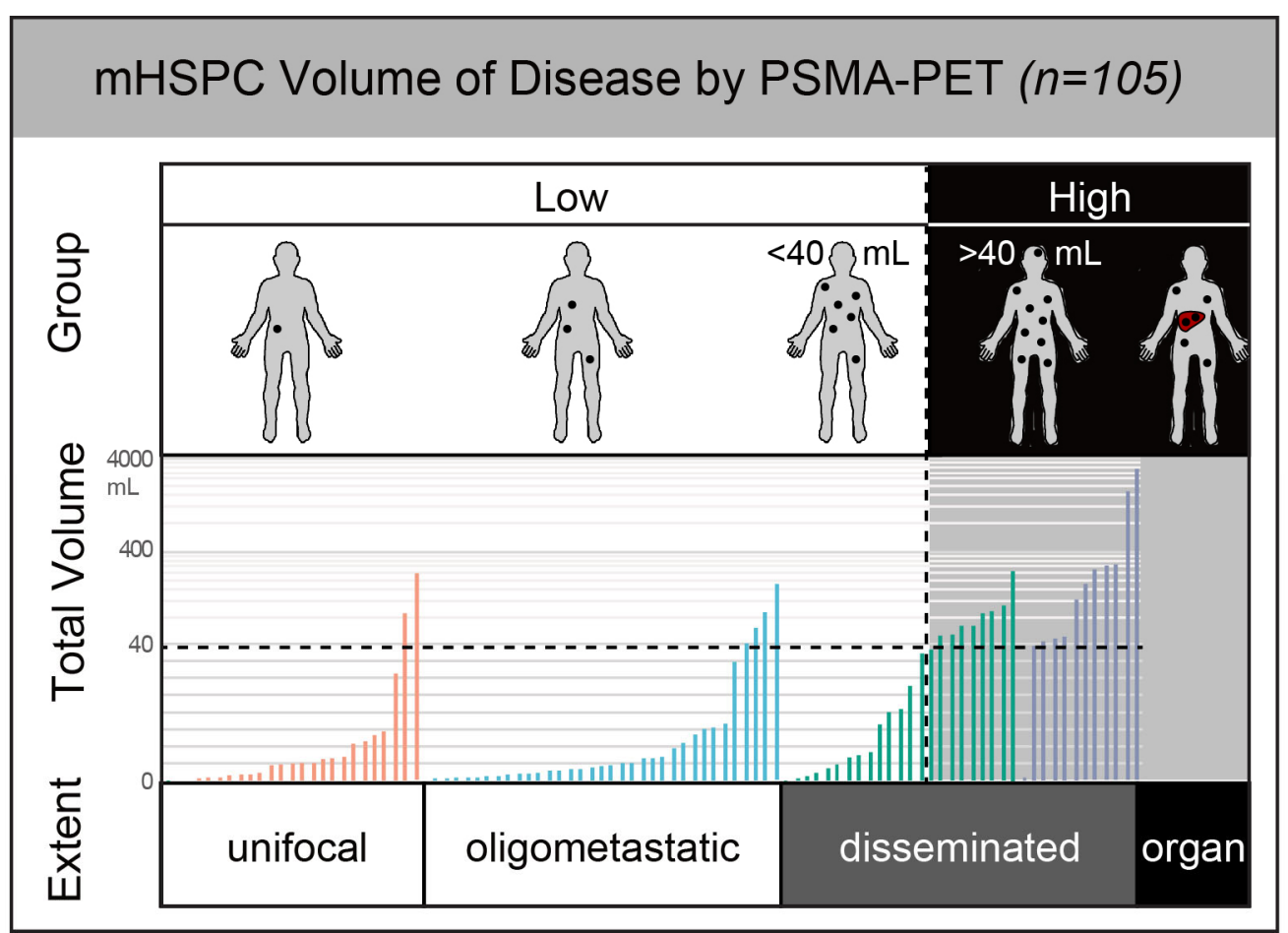


Supplemental Table 1: Patients' characteristics.

\begin{tabular}{|c|c|}
\hline & $\begin{array}{l}\text { All PSMA-PET- } \\
\text { patients }(N=105)\end{array}$ \\
\hline \multicolumn{2}{|l|}{ Age (years) } \\
\hline Median (range) & $68.2(48-84)$ \\
\hline \multicolumn{2}{|l|}{ Prostate-specific antigen $(\mathrm{ng} / \mathrm{mL})$} \\
\hline Median (range) & $2.6(0.1-1764)$ \\
\hline \multicolumn{2}{|l|}{ Gleason score } \\
\hline$<8$ & $43(41)$ \\
\hline$\geq 8$ & $56(53)$ \\
\hline $\mathrm{N} / \mathrm{A}$ & $6(6)$ \\
\hline \multicolumn{2}{|l|}{ Prior therapy } \\
\hline Prior prostate cancer-related surgery & $79(75)$ \\
\hline \multicolumn{2}{|l|}{ Prior prostate cancer-related } \\
\hline radiotherapy & $47(45)$ \\
\hline ADT & $16(15)$ \\
\hline \multicolumn{2}{|l|}{ Conventionally assessed tumor } \\
\hline \multicolumn{2}{|l|}{ burden } \\
\hline LVD & $85(81)$ \\
\hline HVD & $20(19)$ \\
\hline \multicolumn{2}{|l|}{ N/M status by $\mathbf{C T}$} \\
\hline N1 & $5(5)$ \\
\hline M1a & $1(1)$ \\
\hline M1b & $91(87)$ \\
\hline M1c & $8(8)$ \\
\hline
\end{tabular}

Data are number of patients (\%) unless otherwise indicated. 
Supplemental Table 2: mHSPC TNM by computed tomography (CT) versus PSMA-PET.

\begin{tabular}{|c|c|c|c|c|c|c|}
\hline & \multicolumn{7}{|c|}{ PSMA-PET } \\
\hline \multirow{4}{*}{ Stage, n (\%) } & no disease & Tr/N1 only & Any M1a & Any M1b & Any M1c \\
\hline \multirow{2}{*}{ no disease } & $\mathbf{0}$ & 0 & 0 & 0 & 0 \\
\hline Tr $/$ N1 only & 0 & $\mathbf{3}(\mathbf{3})$ & 0 & $1(1)^{*}$ & $1(1)^{*}$ \\
\hline Any M1a & 0 & 0 & $1(1)$ & 0 & 0 \\
\hline Any M1b & 0 & 0 & 0 & $\mathbf{9 0}(\mathbf{8 6})$ & $1(1)^{*}$ \\
\hline & Any M1c & 0 & 0 & 0 & 0 & $\mathbf{8}(\mathbf{8})$ \\
\hline
\end{tabular}

*PET upstaging for 3 of 105 (3\%) patients.

Supplemental Table 3: Comparison of disease extent as stratified by CT-based CHAARTED vs. PET-based miTNM Volume criteria

\begin{tabular}{|c|c|c|c|c|c|c|}
\hline & \multicolumn{6}{|c|}{ PSMA-PET } \\
\hline \multirow{3}{*}{ CT } & uni & oligo & $\begin{array}{c}\text { diss }<\sim 40 \\
\text { ml }\end{array}$ & $\begin{array}{c}\text { oligo/ } \\
\text { diss } \geq \sim 40 \\
\text { ml }\end{array}$ & M1c \\
\cline { 2 - 8 } & $\begin{array}{c}\text { CHAARTED } \\
\text { LVD }\end{array}$ & $25(29)$ & $31(36)$ & $17(20)$ & $\mathbf{1 0 ( 1 2 ) *}$ & $\mathbf{2}(\mathbf{2}) *$ \\
\cline { 2 - 8 } & $\begin{array}{c}\text { CHAARTED } \\
\text { HVD }\end{array}$ & 0 & 0 & $\mathbf{1}(\mathbf{5}) *$ & $11(55)$ & $8(40)$ \\
\hline
\end{tabular}

*Mismatch between CT (CHAARTED) and PET (miTNM VOL) classification 